\title{
Determination of the Whole-Body Absorption Cross Section of a Phantom using RiMAX
}

\author{
Aliou Bamba*, Wout Joseph member IEEE, \\ Emmeric Tanghe, Luc Martens member IEEE \\ Ghent University/iMinds, Department of Information Technology \\ Gaston Crommenlaan 8 box 201, B-9050 Ghent/Belgium \\ Email: aliou.bamba@intec.ugent.be
}

\author{
Davy Gaillot, Martine Lienard \\ Group TELICE, IEMN, University of Lille \\ Building P3, 59655 Villeneuve d'Ascq, France
}

\begin{abstract}
The reverberation time has been measured in a reverberation chamber for different loads and for a cross polarized transceiver at the frequency of $1.8 \mathrm{GHz}$. We determine the whole-body absorption cross section of a canonical phantom using a maximum-likehood high-resolution channel parameters estimator - RiMAX - and good agreement has been obtained with the result from the numerical simulations. The relative error between the simulated values and the RiMAX based measured values is less than $1.3 \%$.
\end{abstract}

Keywords: Reverberation chamber, reverberation time, diffuse multipath components, maximum-likelihood high-resolution channel parameters estimator, whole-body absorption cross section.

\section{INTRODUCTION}

$\mathrm{R}^{\mathrm{n}}$ EVERBERATION chambers have been primarily used for the measurements of the electromagnetic compatibility (EMC). The electromagnetic fields (EMF) are diffuse in a reverberation chamber due to the richness of the environment in multiple paths. This characteristic enhances the performances of the multiple input multiple output (MIMO) channels and the reverberation chambers have been therefore used for the wireless MIMO channels characterization [1]. Reverberation chambers have been also used to calculate the absorption cross section of lossy objects under a plane wave illumination, which is a crucial information when assessing an antenna radiation efficiency located close to lossy object such as a head phantom.

We recently developed a method to determine the whole-body absorption cross section $\left(\mathrm{ACS}_{w b}\right)$ of a in a realistic environment [2] and validated it via numerical simulations in [3]. The $\mathrm{ACS}_{w b}$ determination was based on the room electromagnetics [4] assumption in the investigated environments. The reverberation chamber is the perfect environment wherein the properties of the room electromagnetics theory can be emulated since the theory only supposes one specular component - the Line Of Sight when it exists - the remainder is supposed to be part of the diffuse multipath component (DMC). The method we developed relies on a manual algorithm to determine the room reverberation time and thereby the human $\mathrm{ACS}_{w b}$. An analytical and automatic algorithm which would be more stable and more reliable is important in order to determine accurately the whole-body $\mathrm{ACS}_{w b}$ of a phantom located in the reverberation chamber.

The object is to investigate the $\mathrm{ACS}_{w b}$ of a canonical phantom - through measurements in a reverberation chamber - by using the well known maximum-likelihood high-resolution channel parameters estimator: RiMAX. The paper is organized as follows: we introduce the materials and the methodology in Section II. Section III presents the result of the measurements as well as the simulations result. Finally, conclusions are drawn in Section IV.

\section{Materials AND Methods}

\section{A. Presentation of the reverberation chamber}

A Steered-Mode Reverberation Chamber is a fully-closed metallic cavity which is oversized with respect to the investigated wavelength. It operates over a large frequency bandwidth (1 $18 \mathrm{GHz}$ ) depending upon its dimensions and volume. Due to the cavity effect, there exists a working volume away from the walls wherein the electromagnetic fields are statistically uniform for all measurement points, directions and electromagnetic emitting source positions. The uniformity of the fields is obtained via a rotating mechanical pale inside the chamber that steers the electromagnetic modes.

The measurements were performed at the University of Lille 1 in the TELICE research group reverberation chamber. The reverberating chamber dimensions are $2.8 \mathrm{~m} \times 5.7 \mathrm{~m} \times 4.1 \mathrm{~m}(\mathrm{H} \mathrm{x} \mathrm{L} \mathrm{x}$ 1) and $65 \mathrm{~m}^{3}$ volume.

\section{B. Presentation of the materials and measurement set up}

The study has been carried out at the central frequency of $1800 \mathrm{MHz}$ within a bandwidth of $100 \mathrm{MHz}$.

The measurements have been performed with four cylindrical phantoms in polyvinyl chloride (PVC) located arbitrary in the working area of the reverberation chamber. A phantom has an inner radius of $119.5 \mathrm{~mm}$ and an outer radius of $124.5 \mathrm{~mm}$ (thickness $=5 \mathrm{~mm}$ ) and a height of $1500 \mathrm{~mm}$. We used a tissue equivalent liquid that has similar dielectric properties than the body tissue parameters specified in [5], and filled the phantoms with it. The liquid has a relative permittivity $\epsilon_{r}=57.74$, a conductivity $\sigma=1.47 \mathrm{~S} / \mathrm{m}$ and a mass density of $\rho=1000 \mathrm{~kg} / \mathrm{m}^{3}$. The dielectric properties of the liquid were measured using a Hewlett Packard HP85070A dielectric probe kit with a Hewlett Packard HP8753D network analyzer. As transceivers, two Double Ridge Guide Horn Antennas of type SAS-571 were placed in the reverberation chamber in such a way that there was no line of sight view. Because we want to determine the whole-body $\mathrm{ACS}_{w b}$ in complete diffuse fields, the specular components mainly the LOS component - have to be discarded from the power delay profile (PDP), justifying thereby the antennas cross polarization.

\section{Presentation of the channel parameters estimation algorithm}

Recent studies have shown that the wireless narrow band radio channel $h$ can be understood as a superposition of deterministic specular components $s(\Theta)$ and stochastic dense multipath components $d\left(\Theta_{d m c}\right)$, which include diffuse components [6]. Here, 
$\Theta_{s c}$ and $\Theta_{d m c}$ are the set of parameters that fully describe the propagation mechanisms.

$$
h=s\left(\Theta_{s c}\right)+d\left(\Theta_{d m c}\right)
$$

The sampled response vector $h \in \mathbb{C}^{M_{f} \times 1}$ (where $M_{f}$ corresponds to the number of frequency points) is assumed to follow a multivariate circular symmetric complex Gaussian process with mean $s\left(\Theta_{s c}\right)$ and covariance $R\left(\Theta_{d m c}\right)$ :

$$
h \approx N_{c}\left(s\left(\Theta_{s c}\right), R\left(\Theta_{d m c}\right)\right)
$$

As it can be seen in Fig. 2-4 of [6], the DMC power $\psi(\tau)$ as function of time delay $\tau$ is described by an exponential decay:

$$
\phi(\tau)=\alpha_{1} e^{-\beta_{d}\left(\tau-\tau_{d}\right)}+\alpha_{0}
$$

In (1), $\alpha_{1}, \beta_{d}, \tau_{d}$ and $\alpha_{0}$ are the four parameters which fully describe the DMC and are gathered into the DMC parameter vector [6]. This parametric description of the channel enables the use of maximum-likelihood high-resolution channel parameter estimation algorithms, such as RiMAX.

The first step is the determination of $\beta_{d}$, which is the slope of the decaying tail of PDP. Note that $\beta_{d}$ is the inverse of the reverberation time. Since the reverberation time of the chamber is obtained by processing the measured data with the RiMAX algorithm, we applied the same method as in [2], [3] to determine the whole-body absorption cross section of the cylindrical phantom. Furthermore, we determined the whole-body $\mathrm{ACS}_{w b}$ of the cylindrical phantom through numerical simulation for validation purpose. The Finite Difference Time Domain (FDTD) solver SEMCAD-X is used to compute numerically the whole-body $\mathrm{ACS}_{w b}$ of the phantom from its whole-body specific absorption rate $\left(\mathrm{SAR}_{w b}\right)$ (Section $\mathrm{D}$ of [2]). The plane waves are assumed to be in the horizontal plane (no elevation) in the simulation tool. The same dielectric properties used in the reverberation chamber are used in the simulation settings. The numerical whole-body absorption cross section of the cylindrical phantom is comprised of the ACS of the cylinder contour and the ACS of the cylinder's top since these are the exposed surfaces in the reverberation chamber.

\section{RESULTS}

For the experimental measurements, we loaded the reverberation chamber with $0,1,2, \ldots, 4$ phantom(s) filled with the tissue equivalent liquid and we determined in each case the related PDP. Some PDP graphs are shown in Fig. 1. For each PDP, the

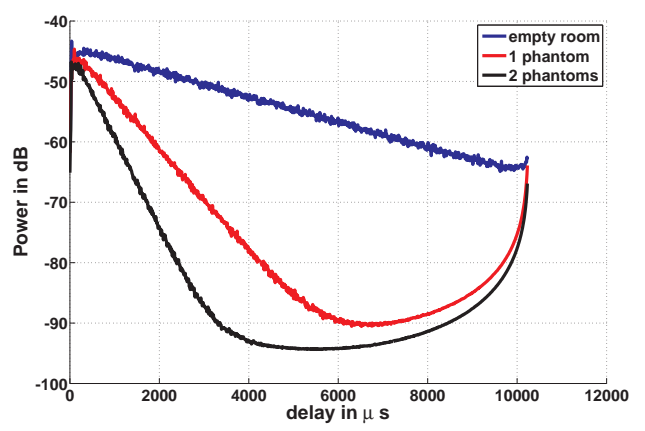

Fig. 1. Power Delay Profiles in the reverberation chamber (cross polarization)

reverberation time is determined using the RiMAX estimator. The result in terms of the reverberation time is shown in Fig 2. It is clear from Figs 1, 2 that adding a load in the chamber changes its

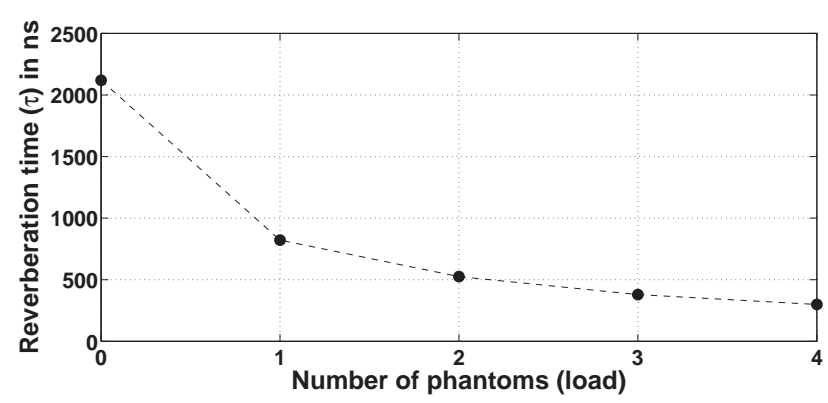

Fig. 2. Reverberation time values for different phantoms in the reverberation chamber

properties. The highest value of the reverberation time is obtained in the empty room whereas the lowest value is obtained when the chamber has the maximum load, i.e., 4 phantoms. The presence of a phantom in the chamber increases the absorbing area of the EMF, causing a fast decrease of the energy in the chamber which in turn results in a low reverberation time.

Knowing the reverberation time values for the different loads, we used the methodology in [2] to determine the whole-body $\mathrm{ACS}_{w b}$ of a phantom. We obtained an experimental whole-body $\mathrm{ACS}_{w b}$ of $0.621 \mathrm{~m}^{2}$ whereas a whole-body $\mathrm{ACS}_{w b}$ of $0.629 \mathrm{~m}^{2}$ is obtained from the numerical simulation, leading to a relative error of $1.27 \%$.

\section{CONCLUSIONS}

We determine the whole-body absorption cross section $\left(\mathrm{ACS}_{w b}\right)$ of a phantom in a reverberation chamber using the RiMAX estimator. The numerical simulations result of the same phantom has shown that the high resolution algorithm RiMAX gives an excellent estimation of the phantom whole-body $\mathrm{ACS}_{w b}$ since a relative error of $1.27 \%$ is obtained. Future research will consist of the determination of the whole-body absorption cross section of a human for different postures, which is useful for human specific absorption rate assessment in a realistic environment.

\section{REFERENCES}

[1] M. Liénard and P. Degauque. Simulation of dual array multipath channels using mode-stirred reverberation chambers. Electronics Letters, 40(10): pages 578-580, 2004.

[2] A. Bamba, W. Joseph, J. B. Andersen, E. Tanghe, G. Vermeeren, D. Plets, J. O. Nielsen, and L. Martens. Experimental Assessment of Specific Absorption Rate Using Room Electromagnetics. IEEE Transactions on Electromagnetic Compatibility, 54 (4): pages 747-757, 2012.

[3] A. Bamba, W. Joseph, G. Vermeeren, E. Tanghe, D. P. Gaillot, J. B. Andersen, J. Ø. Nielsen, M. Lienard and L. Martens. Validation of Experimental wholebody SAR Assessment Method in a Complex Environment. Bioelectromagnetics, 34 (2): pages 122-132, 2013.

[4] J. Bach Andersen, J. Ø. Nielsen, G. F. Pedersen, G. Bauch, and M. Herdin. Room Electromagnetics. IEEE Antennas and Propagation Magazine, 49, No. 2: pages 27-33, April 2007.

[5] D. L. Means and K. W. Chan. Evaluating Compliance with FCC Guidelines for Human Exposure to Radiofrequency Electromagnetic Fields. Technical report, Federal Communications Commission Office of Engineering \& Technology, 2001.

[6] A. Richter. Estimation of Radio Channel Parameters: Models and Algorithms. $\mathrm{PhD}$ thesis, Faculty of electrical Engineering and Information Technology at the Technical University. Ilmenau, Germany., 2005. 